\title{
A Categorization Theory of Spatial Voting: How the Center Divides the Political Space
}

\author{
Jørgen Bølstad \\ Center for Comparative and International Studies, Swiss Federal Institute of Technology Zurich \\ (email: joergen.boelstad@eup.gess.ethz.ch) \\ Elias Dinas \\ Department of Politics and International Relations, University of Oxford \\ (email: elias.dinas@politics.ox.ac.uk) \\ Forthcoming in British Journal of Political Science
}

\begin{abstract}
We present a categorization theory of spatial voting, which postulates that voters perceive political stances through coarse classifications. Because voters think in terms of categories defined by the ideological center, their behavior deviates from standard models of utility-maximization along ideological continua. Their preferences are characterized by discontinuities, rewarding parties on their side of the ideological space more than existing spatial models would predict. While our analysis concurs with prior studies suggesting that voters tend to use a proximity rule, we argue that this mainly serves to distinguish among parties of the same side. Overall, our findings suggest that voters' party evaluations are characterized by a nontrivial identity component, generating in-group biases not captured by the existing spatial models of voting.
\end{abstract}

Center for Comparative and International Studies, Swiss Federal Institute of Technology Zurich (Email: joergen.boelstad@eup.gess.ethz.ch); Department of Politics and International Relations, University of Oxford (Email: elias.dinas@politics.ox.ac.uk). The authors would like to thank James P. Cross, Spyros Kosmidis, Jonathan Nagler, Trajche Panov, Pedro Riera, Piero Stanig, Alex Trechsel, Pablo Fernandez-Vazquez, Till Weber, Thomas Winzen, the members of their panels at the 2nd Annual General Conference of the European Political Science Association, Berlin, 21-23 June 2012, and the Annual Elections, Public Opinion and Parties Conference, at the University of Exeter, 9-11 September 2011, as well the attendants of relevant sessions of the ETH Colloquium on European Politics, and the EUI Colloquium on Political Behavior. Replication codes as well as an online appendix with supplementary information are available at http://dx.doi.org/doi: 10.1017/S0007123415000393. All data used in this article are publicly available. The main dataset is the European Election Study 2009, available at https://dbk.gesis.org/dbksearch/sdesc2.asp?no=5055\& $\mathrm{db}=e \& d o i=10.4232 / 1.10202$. 
Politics is about sides. It is about knowing your friends and your enemies; it is about groups, sharing goals and values. The most general groups are defined by their side of the center. We thus speak of liberal and conservative voters; left- and right-wing parties. ${ }^{1}$ Commentators do so as a matter of course and it would be strange if voters were any different. Yet, none of the existing theories of spatial voting capture this logic. The dominant proximity theory simply posits that voters will prefer the party closest to themselves. ${ }^{2}$ The alternative directional theory $^{3}$ divides the political space into two directions of policy change, but, as we show here, its theoretical underpinnings are substantively different and it consequently fails to capture the effect we hypothesize.

We offer a categorization theory of spatial voting, starting from the observation that individuals simplify choices by classifying objects into categories. ${ }^{4}$ Several lines of research have shown that categorization tends to influence how objects are perceived. In particular, individuals tend to underestimate the differences between observations falling in the same category and exaggerate the differences between those falling in different categories. ${ }^{5}$ Social identification theory extends this idea to social categorizations, holding that individuals derive their identities from group memberships and therefore exaggerate the differences between group insiders and outsiders. ${ }^{6}$ We argue this also applies to spatial voting: Ideological categorization is not only an information shortcut; self-categorization is also an expression of identity.

Our resulting model of spatial voting generates a set of testable predictions, which qualify the theoretical foundations of existing spatial theories. First, rather than just operating as

1 Employing European data, we focus on parties, but we might as well have presented our argument in terms of individual candidates in a two-party setting. We report an analysis of American data in the On-line appendix.

2 See ??.

3 ?.

4 See ???.

5 ?.

$6 ?$. 
ideal-point seekers along continuous policy dimensions, voters form preferences over coarse categories. Voters' loss functions therefore also reflect whether parties fall into the same broad category as themselves. Parties falling in the same category as the voters receive a bonus, beyond what would be predicted by other spatial theories.

Second, when voters have multiple parties on their side, they are forced to apply more specific criteria. No such decision rule is needed for parties on the opposite side. Thus, although categorization theory is agnostic about the exact criterion used to choose between parties on a given side, it does anticipate that this criterion will be more important in distinguishing between options within the preferred category. In our analyses, we find a clear proximity pattern in addition to categorization effects, and we would expect this effect to be stronger for parties on the same side as voters. In this sense, our theory does not replace the existing ones; it rather refines and qualifies them, by adding a component that has so far been overlooked.

Third, voters' efforts to rationalize non-ideological party preferences by letting these influence their perceptions of party positions - a phenomenon known as projection bias - should also be characterized by a category-based discontinuous pattern. The drive to exaggerate a preferred party's proximity to one's own ideological views will be stronger when doing so also implies placing it on one's own side of the center.

Our empirical analyses test all these implications of the theory. Before we delve into the tests, however, we elaborate on the theoretical underpinnings of categorization. We begin by presenting the main theories of spatial voting, but only briefly, given the vast existing literature. We then introduce our categorization theory and show how other models fail to capture the effects we predict. This is followed by a set of empirical analyses and robustness checks. The conclusion elaborates on the implications of our findings. 


\section{Existing Theories}

Spatial theories of voting share the assumption of rational voters choosing the alternative that gives them the highest utility based on spatial considerations. They differ, however, in the utility functions that translate the policy positions of voters and parties into utility losses. The most straightforward model of vote choice, the "proximity model," holds that individuals vote for the parties whose positions are the most similar to their own. ${ }^{7}$ For a given political dimension, we calculate the Proximity Term for voter $i$ and party $j$ as the absolute distance between the position of the voter $\left(v_{i}\right)$ and the position of the party as perceived by the voter $\left(p_{i j}\right)$, i.e. $\left|v_{i}-p_{i j}\right| \cdot{ }^{8}$ This term is expected to have a negative coefficient, as it captures distances that voters are expected to penalize. The utility curve implied by the model peaks where voters and parties are at the same position. This is shown in the left panel of figure ??, which displays the utility for voters located at -2 , and +1 , on a dimension from -5 to +5 as a function of a party's position along the same dimension.

? present a competing "directional model," drawing on earlier work on issue perception and symbolic politics. ${ }^{9}$ The authors argue the assessment of political parties or candidates reflects two considerations. The first is "whether the individual and candidate are in agreement about the direction public policy should take". ${ }^{10}$ If individuals and parties prefer the

$7 ?$.

8 An alternative to the absolute or "city-block" distances between $v_{i}$ and $p_{i j}$ is to use squared "Euclidean" distances, i.e. $\left(v_{i}-p_{i j}\right)^{2}$. However, this alternative tends to give a worse fit than absolute distances. In a study of candidate ratings, for example, ? (?, 283) notes that "the linear proximity function outperforms the quadratic function in all cases ... suggesting that the linear form may be preferable" (see also ?, 24, fn. 5). As can be seen in figures ?? and ?? below, this is also the case here. Therefore, we focus on the specification with absolute distances. It should be noted, however, that we also report analyses including factorized proximity scores, i.e. fixed effects, thus controlling for any proximity-based function including the squared version, and our results still hold. We also return to this issue in the conclusion.

9 ??

$10 ?, 96$. 


\section{Figure 1: Voter Utilities according to the Proximity and Directional Model}
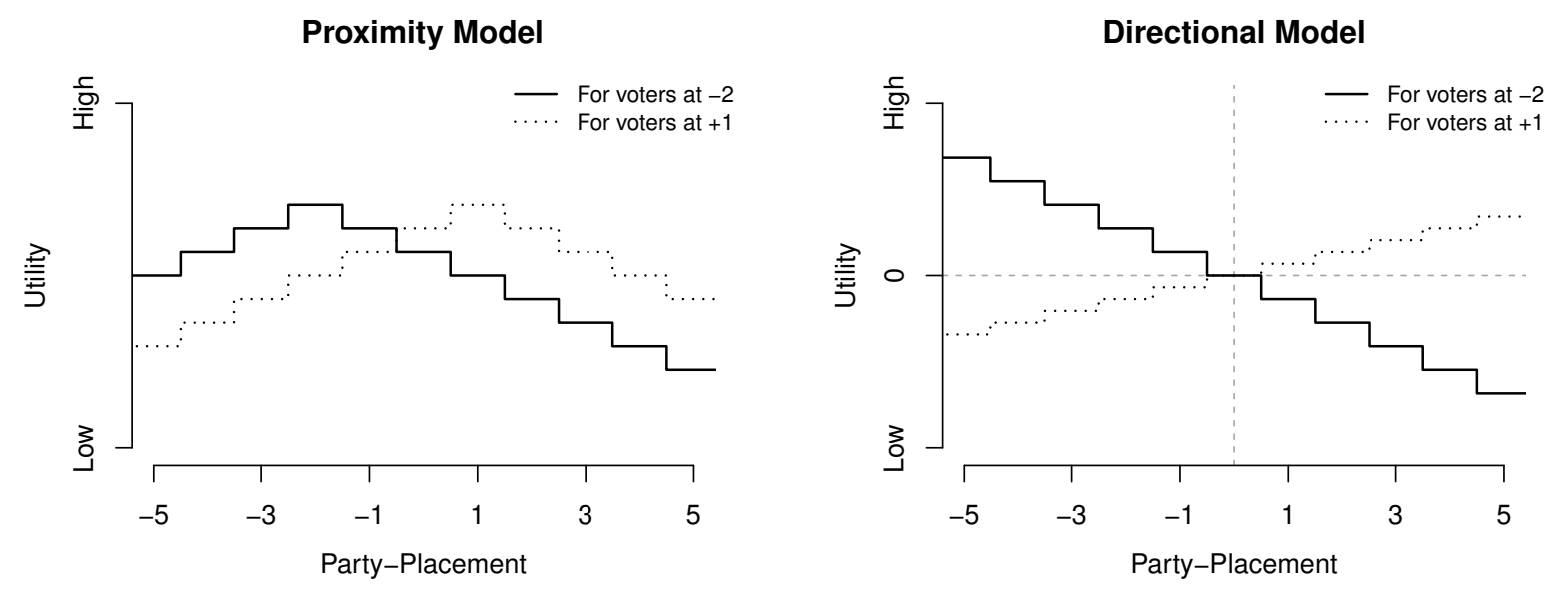

same direction, this will contribute positively to party assessment, if they do not, the contribution will be negative. Second, the authors argue that the strength of the contribution to party assessment will be an interaction of the intensities with which the voter and party is emphasizing a given direction: The further they both are from the "neutral point," the stronger the response. The neutral points stands for the point at which no desired policy change is implied, and is commonly operationalized as the center of the scale. ${ }^{11}$

Following ?, we thus calculate the Directional Term as $v_{i} \times p_{i j}$, where the notation is the same as above, and $v_{i}$ and $p_{i j}$ are centered on the middle of the scale. As shown in the right panel of figure ??, for a voter at a given position, the directional utility of voting for a party is a linear function of the party's position. If the voter is on a given side, the utility will increase linearly as the party moves towards that side, crossing 0 when the party crosses the center. The role of the voter position is to change the slope of the function: The further the voter moves from the center, the steeper the slope. The same holds for party positions, of course: They change the slope of the utilities as a function of voter positions. ${ }^{12}$

${ }^{11}$ We discuss the concept of the neutral point and the issue of operationalization in more detail in the On-line Appendix (Section A.5).

12? also qualified their model, suggesting that parties located outside the region of acceptable policy platforms will be punished by directional voters for being too extreme. However, as ? (?) has noted, it is problematic to conceptualize the region of acceptability within a directional framework: The idea that parties are too extreme is inherently based on a 
Both in theoretical discussions and empirical studies, the intensity aspect of directional theory has overshadowed the directional aspect. The motivation behind most work in this area has been to solve the puzzle of the "empty center:" The question of why parties and candidates appear to be more extreme than voters. ${ }^{13}$ This emphasis on the intensity part of the model has also motivated the accumulation of numerous mixed models of spatial voting. Perhaps most important among these is Grofman's discounting model, which has been further elaborated in subsequent work. ${ }^{14}$ The key idea is that parties are unable to fully implement their proposals, due to within-party dynamics ${ }^{15}$ or due to systemic constraints in multiparty systems with coalition governments. ${ }^{16}$ Thus, voters are led to prefer parties more extreme than themselves to get the amount of policy change they would like. These models are all based on a proximity calculus, adjusted to accommodate the discrepancy between parties' programmes and their actual policies. ${ }^{17}$ We leave these additional models aside here, as they do not capture the effects we hypothesize to any greater extent than the two main theories that we focus on.

In addition to the development of mixed models, a vast literature has emerged, trying to assess the relative merits of the proximity theory and the directional theory. ${ }^{18}$ ? argue, however, that arguments about which theory fits best tend to be based on assumptions that

proximity logic. Furthermore, few empirical studies have taken this idea into account. Even the originators of directional theory abandoned this idea in their more recent research. See ??. We thus leave this issue aside here.

$13 ?$.

$14 ? ?$.

$15 ?$.

$16 ?$.

17 ???. ? (?) follow the alternative strategy of constructing a "unified" model, encompassing a squared proximity term, as well as directional components, separating the intensity part and a purely directional part. The authors trace the purely directional component back to ? (?), and implement it as the cosine of the angle between voter and candidate.

${ }^{18}$ See ?????. 
are hard or impossible to test. ${ }^{19}$ Recently, several experiments have brought the debate forward, concluding overwhelmingly in favor of proximity theory. ${ }^{20}$ Overall, there is little doubt that proximity theory has considerable predictive power in voting behavior. We argue, however, that the existing theories of spatial voting, including proximity theory, overlook an important pattern, reflecting categorization effects.

\section{Categorization Theory}

Research in cognitive science has repeatedly demonstrated that humans use coarse perceptual schemas that simplify information processing. ${ }^{21}$ Under conditions of uncertainty, categorization of objects permits the use of simple heuristics to make predictions regarding unknown object features. ${ }^{22}$ Work in judgment theory in social psychology has further shown that merely placing objects in categories influences individuals' perceptions and evaluations of these objects. ${ }^{23}$ This form of bias is known as accentuation and involves a tendency for individuals to underestimate the differences between observations falling in the same category and to overestimate the differences between those falling in different categories. ${ }^{24}$ Furthermore, social identification theory holds that such effects are involved in the forma-

19 ?. See also ?.

$20 ? ? ?$.

$21 ? ? ?$.

22 ??. Economists have formalized this process showing how coarse thinkers differ from the standard Bayesian updating prototype and further illustrated the implications of categorical thinking for stock market pricing and advertising. See ??.

$23 ? ?$.

24 ?????. A study by ? (?) illustrates such effects nicely. When asked to estimate the temperature in Providence, Rhode Island, subjects showed no general pattern of over- or under-estimation. However, they showed a remarkable tendency to shift their estimates disproportionally when the month changed. Thus, between two equally spaced days, the average difference in estimated temperatures was smaller when both days were in same month than when they were in adjacent months. As noted by ? (?, 7), the intuitive explanation for this pattern is that the subjects used the month as a category of reference in their estimates. 
tion of social identities, as individuals not only categorize objects, but also people, including themselves. ${ }^{25}$ Because individuals partly derive their identities from self-perceived group memberships, they tend to attach strong feelings to such memberships, which in turn leads them to focus on, and exaggerate, the differences between group members and outsiders. ${ }^{26}$ In other words, identity formation is based on self-categorizations which in turn leads to in-group favoritism. ${ }^{27}$

We argue that these findings are relevant to spatial voting, because voters operate with basic mental categories representing the sides of the political center. In particular, this applies to the most important political dimension in a given political system, yielding two encompassing categories. In most countries, it is common to speak of voters and parties as being either left-wing or right-wing, and these labels provide shortcuts to richer descriptions of the actors' traits. $^{28}$ As with other stimuli, the categorization of political actors according to their side of the ideological center is likely to involve the use of prototypes. ${ }^{29}$ A prototype left-wing actor may, for example, emphasize social and economic equality and express a willingness to use public policy to promote these values, while a right-wing actor may emphasize individual responsibility and freedom from government intervention. Thus,

$25 ? ?$.

${ }^{26} ? ?$.

${ }^{27}$ See ?. While categorization effects have generally received little attention in political science, in-group favoritism has been recognized as a key component of social-identity accounts of party identification. See ???. Here, however, we focus on spatial categorizationbased biases and we look at larger categories than parties themselves. We discuss the issue of party identification further below, as well as in the robustness section.

${ }^{28}$ In the American setting, actors are similarly categorized as liberal or conservative. However, the distinction between parties and ideological sides might be less relevant in a two-party system, as candidates tend to self-select into one of the two parties, and those who significantly deviate from the party's ideological label are punished by voters. See ?. Nevertheless, we argue that ideological categorizations and party-based classifications are different phenomena, and that categorization is relevant also to understand American voting behavior. We report analyses testing these arguments in the robustness section and the On-line Appendix.

${ }^{29}$ ???? 
categorization is also accompanied by issue packaging, permitting the use of coarse party preferences as a heuristic. Even if specific issue dimensions might allow refined distinctions between parties, they are eventually embedded within a coarse, category-based, assessment framework. Theories of party branding exemplify this logic, suggesting that voters perceive parties' placements along salient dimensions as signals of what they generally stand for. ${ }^{30}$

Drawing on these arguments, we expect voters to assess and be conscious of their own side of the ideological center. As ideological positions tend to be highly consistent over time ${ }^{31}$ we further assume that these self-categorizations matter for voters' identities, expressing some of their most fundamental values and attitudes. As a consequence, we expect voters to assess whether a given party falls in the same category as themselves - in other words, whether the party is on their side, being one they broadly agree with and potentially could vote for. The first empirical implication of our theory is therefore that parties receive a more favorable assessment when they are on the same side as the voter in question. Furthermore, allowing for the existence of a neutral center category, we expect voters to penalize parties on the opposite side, more than those in the center. As illustrated in the next section, these effects are not captured by the dominant theories of spatial voting.

In a setting with many parties, however, a pure categorization model is unlikely to be adequate on its own, as voters may still have to choose among several parties on their side. Existing research suggests that individuals facing a large number of options indeed are likely to first choose a broad category, and then make a more specific choice within this category. ${ }^{32}$ In the present context, such a within-category choice could be based on either

${ }^{30}$ See ?. The dynamics of early political socialization also facilitate the presence of categorization effects. Parental transmission of ideological inclinations does not operate through policy-driven orientations. It rather consists of a learning process, which is centered around easily recognized labels: left versus right, liberal versus conservative; or nationalist versus cosmopolitan. See ?. Children do not learn parents' ideal points along issue dimensions, but form beliefs regarding broad ideological categories, which often carry significant historical connotations. See ?.

${ }^{31} ?$.

$32 ?$. 
of many available decisions rules. Voters could make a random draw, choose based on nonideological factors (e.g. experience, charisma, and competence), or conduct a more finely grained assessment among parties on their side, applying additional functions of party and voter positions. This last scenario is particularly interesting because it implies that different spatial rules may interact with categorization effects. While voters need to pick a favorite to win their vote, it matters less how they rank their least favored parties. Thus, according to our theory, the predictive power of existing spatial rules should increase when categorization is insufficient to distinguish among the alternatives, i.e. for parties located on the same side of the ideological spectrum. If, for example, voter use a proximity rule, we expect that its impact on vote choice increases when parties and voters are on the same side.

Furthermore, the argument that voters think in terms of categories has implications for the issue of reverse causation from preferences to party perceptions. A common challenge to analyses of proximity voting relates to a phenomenon known as projection or rationalization bias, which refers to the tendency for voters to exaggerate their ideological agreement with their preferred political parties. ${ }^{33}$ Given the utility maximization principle of spatial models, this endogenous process complicates the identification of ideological voting. In the current setting, however, the issue of reverse causation also offers an additional opportunity to test our theory, which seeks to describe the lenses through which voters understand politics. While it is possible that some voters bring parties they like over to their side to justify their preferences, this would only confirm that voters do think in terms of these broad categories. In fact, if our theory is correct, we should observe categorization patterns of projection bias. We test this implication in the analyses below, and - as a follow-up in the robustness section - we also ensure that our results are not entirely driven by projection, by using objective party-placements. ${ }^{34}$

${ }^{33}$ See, for instance, ?.

${ }^{34}$ Another, more subtle, implication of categorization involves the meaning attached to the middle-category of survey scales. In proximity theory, this midpoint plays no significant role. Directional theory, however, assumes the middle category represents the status quo 


\section{Specification}

If voters indeed do consider whether parties are on their side or not, then some steps along the ideological scale will be more significant than others. In particular, we expect party preferences, as functions of party and voter placements, to exhibit discontinuities at the center of the scale. The expected effects of being on the same and opposite sides are most easily illustrated keeping either voter or party positions fixed. Figure ?? thus illustrates the expected pattern for voters who place themselves left of center.

Assuming the existence of a neutral category, we allow the effect of being on opposite sides to be different from that of being on the same side. In our parametric analyses, we therefore employ two dummy variables to capture the states of relevance. Indicator $S$ captures Same Side status: $S=\left\{1\right.$ if $v_{i} \times p_{i j}>0 ; 0$ if $\left.v_{i} \times p_{i j} \leq 0\right\}$, while indicator $O$ captures Opposite Side status: $O=\left\{1\right.$ if $v_{i} \times p_{i j}<0$; 0 if $\left.v_{i} \times p_{i j} \geq 0\right\}$, where the voter and party positions $\left(v_{i}\right.$ and $p_{i j}$ ) are centered on the geometric middle of the scales. Implementing the categorization model as a regression equation, we get:

$$
Y_{i j}=\beta_{0}+\beta_{1} S_{i j}+\beta_{2} O_{i j}+\varepsilon_{i j}
$$

where $\beta_{0}$ denotes the average preference in the neutral category, $\beta_{1}$ and $\beta_{2}$ are the effects of same and opposite side status, and $\varepsilon_{i j}$ is an error term.

As mentioned, in the presence of multiple parties on the same side as the voter, categorization is not sufficient to distinguish between alternatives. Additional spatial rules may be thus employed by voters to select among these parties. Following this logic, we expect alternative utility functions to be more important in distinguishing among parties on the

that defines directions of policy change. See ????. Categorization theory, on the other hand, holds that the middle-point of the scale denotes the ideological center, separating the sides of the ideological spectrum. According to our argument, voters do not use the midpoint of issue dimensions to denote preferences over policy change but rather as a threshold of coarse ideological categories. We elaborate on these issues and test the two competing conceptualizations in the On-line Appendix. 
same side as the voters. To incorporate this expectation in our model, equation (1) needs to be augmented by the inclusion of appropriate interaction terms, allowing the impact of other functions to depend on categorization:

$$
\begin{aligned}
Y_{i j}=\beta_{0} & +\beta_{1} S_{i j}+\beta_{2} O_{i j}+f\left(v_{i}, p_{i j}\right) \\
& +\beta_{3} S_{i j} f\left(v_{i}, p_{i j}\right) \\
& +\beta_{4} O_{i j} f\left(v_{i}, p_{i j}\right)+\varepsilon_{i j}
\end{aligned}
$$

where $f\left(v_{i}, p_{i j}\right)$ is an unspecified function providing further distinctions. If voters were to apply a proximity logic based on absolute distances, we would get a pattern like that in the left panel of Figure ??, while a directional logic would yield a pattern like that in the right panel. For now, we remain agnostic about which other functions voters may apply. Whichever additional function they apply, however, we expect its effect to be stronger when voters and parties are on the same side.

The empirical analysis is divided into five sections. First, we present a set of illustrative plots, making no assumptions about $f\left(v_{i}, p_{i j}\right)$. We thus explore the presence of categorization effects examining specific scenarios of party and individual ideological placements, without imposing a specific spatial utility function. These results clearly speak in favor of a combination of categorization and proximity effects. Our next step includes a more tailored analysis, aiming at testing the presence of categorization effects when proximity is held constant, while directional theory predicts a different outcome. Next, we put equation (2) to the test in order to investigate a corollary of categorization effects, namely that other spatial criteria will weigh more within the subspace characterized by voter-party convergence. Then, we address the presence of categorization in projection bias, before the last section reports a set of robustness checks. 
Figure 2

(a) Pure Categorization Model

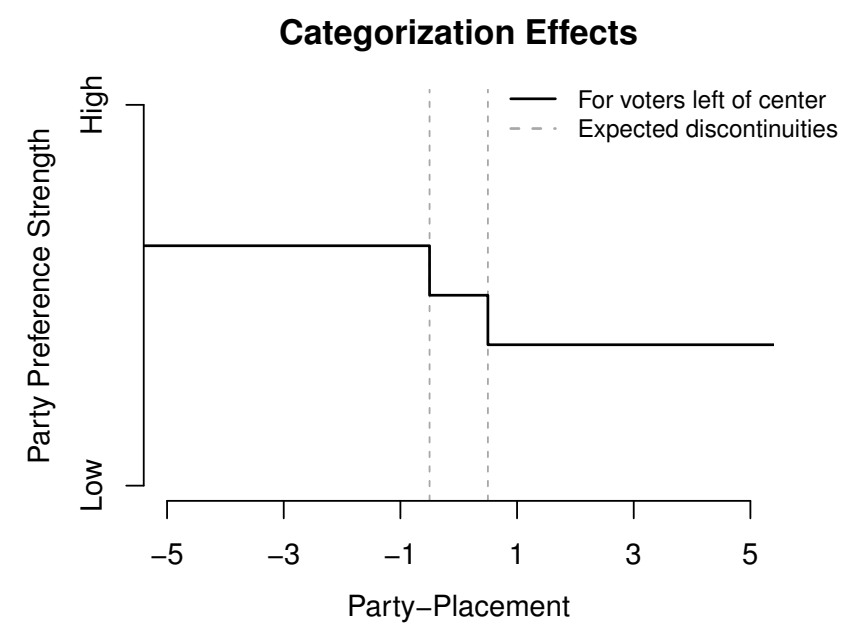

(b) Categorization Effects Conditioning Proximity and Directional Effects
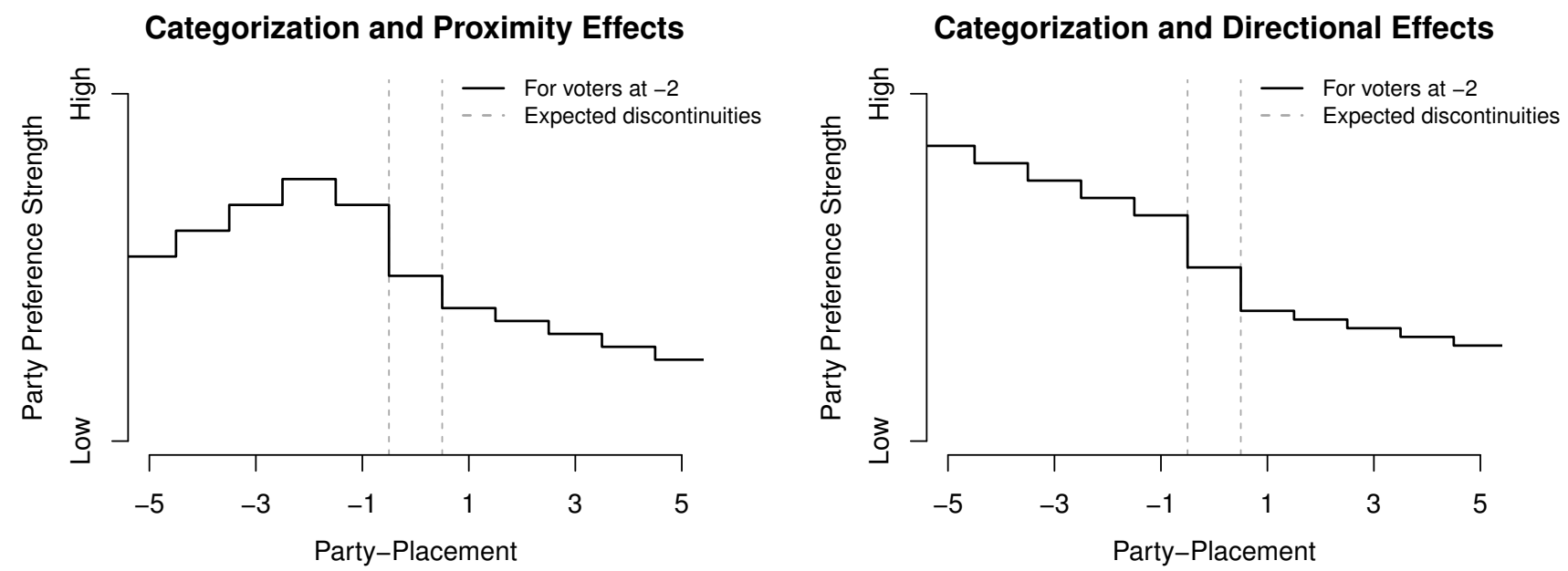


\section{Data and Operationalization}

To test the role of categorization as a general feature of spatial voting, we use data from the European Election Study (EES), ${ }^{35}$ which covers a wide range of countries. We focus on the dominant ideological dimension in these countries, which is the left-right continuum. The EES includes 11-point measures of left-right positions (scales from 0 to 10) for both voters and parties. ${ }^{36}$ To ensure homogeneity in the meaning attached to this scale, we concentrate on established democracies. ${ }^{37}$ We thus base our analysis on $15 \mathrm{EU}$ countries: Austria, Belgium, Denmark, Finland, France, Germany, Greece, Ireland, Italy, Luxembourg, Netherlands, Portugal, Spain, Sweden, and the United Kingdom.

We measure strength of preferences by using the respondents' ratings of the parties, expressed as their likelihood of ever voting for them. This "propensity to vote"-question (PTV) facilitates cross-national comparison and is often used to capture voters' party preferences. ${ }^{38}$ Our unit of analysis is neither voters, nor parties, but rather combinations of voters and parties. We thus transform our dataset to one consisting of party $\times$ individual observations.

We expect voters to use category 5 on the 11-point scales as a neutral category defining two broader categories on each side. To ease the construction and interpretation of the relevant terms, we center the scales on 5 , giving them a minimum of -5 and a maximum of +5 . It is important to note, however, that the survey questions and answer categories do not in any way involve a neutral point, middle, or sides defined by a threshold. They are presented as continuous scales that would seem more for fit employing a pure proximity logic. Thus, if voters are found to understand such a scale in terms of broader categories,

$35 ?$.

${ }^{36} 11$-point scales have been found to have higher validity than other left-right measures. See ?. The On-line Appendix reports the actual wording of all questions used in the analyses. Information about the surveys is also provided (Section A.1).

${ }^{37} \mathrm{~A}$ voluminous literature has emphasized the differences in the content attached to ideological labels in new democracies. See, for instance, ??.

${ }^{38}$ See ?. 
without being asked to do so, this will be valuable evidence against a pure proximity logic.

\section{Visual Inspection}

Choosing to be agnostic about the possible additional utility functions voters apply, we begin with a visual exploration, avoiding parametric restrictions. Our plots are based on local sample means, conditional on party and voter placements. As discussed above, keeping voter positions fixed, the status of being on same or opposite sides becomes a deterministic function of party positions, and vice versa. As explained, we would expect discontinuities on each side of the center. Furthermore, we would expect other spatial rules to have more pronounced effects when parties and voters are on the same side.

Figure ?? shows average party preferences over different voter positions, for each relevant party placement on the left-right scale. For example, the upper left graph of the figure presents the average preference score for a party located at point -1 (left of center) over the whole range of voter positions in the left-right axis, while the lower-left graph shows the same for a party at +1 (right of center). As the figure shows, for almost all party placements there are clear discontinuities as we move from one side of the center to the other, and as explained above, these would not be expected based on a pure directional or proximity model. However, the discontinuities do come in addition to a pattern of finer distinctions, which clearly resemble a proximity model with absolute distances. Furthermore, this pattern is stronger when parties and voters are on the same side: The plots are consistent with the hypothesis that these finer distinctions are conditional on categorization.

Figure ?? shows the same as figure ??, but keeping voters fixed rather than parties. Again, we see categorization effects, albeit somewhat weaker than in the previous plot. Furthermore, the conditioning effect of categorization can be seen also here. When it comes to parties at the opposite side, voters are more indifferent and their preference scores are less contingent upon the smallest distance criterion. These initial analyses thus offer illustrative evidence for the presence of categorization effects. The next sections extend this evidence 
by engaging in a more systematic testing of the constituent parts of the theory.

\section{Critical Cases}

To strengthen the inference that categorization matters, we also conduct more formalized tests, carefully selecting the observations to be compared. In order to distinguish our model from the directional and the proximity models, we focus on so-called "critical cases", ${ }^{39}$ where the three models produce clearly diverging predictions. We conduct four tests, each involving a pair-wise comparison of two scenarios. We thus have eight scenarios, each involving one voter position, and two party positions.

As illustrated in figure ??, the "treatment" scenario of the first comparison $\left(1_{T}\right)$ involves a voter $(V)$ at -1 , a party $\left(P_{A}\right)$ at -3 , and another party $\left(P_{B}\right)$ at $1 .{ }^{40}$ The parties are at an equal distance from the voter, so the proximity model does not predict the voter to prefer one party over the other. According to directional theory, however, $V$ should prefer $P_{A}$ : The directional scores are 3 and -1 for party $A$ and $B$, so the difference between them is 4 (in $A$ 's favor). The respective "control" scenario $\left(1_{C}\right)$ is generated by moving all positions two steps left on the scale. According to proximity theory, the voter should still be indifferent. According to directional theory, however, things are now much more clear-cut: The directional values are 15 and 3 for party $A$ and $B$, respectively. While the difference in directional scores for $P_{A}$ and $P_{B}$ was 4 in $1_{T}$, it is 12 in $1_{C}$. If directional theory is correct, we should expect $V$ to prefer $P_{A}$ to $P_{B}$ to a higher degree in the second rather than the first scenario.

Our categorization model produces a different prediction. In scenario $1_{T}$, party $A$ is on the same side as the voter, while party $B$ is on the opposite side. In scenario $1_{C}$, both parties are on the same side as the voter. Hence, $V$ should prefer $P_{A}$ over $P_{B}$ to a greater extent in scenario $1_{T}$ than in $1_{C}$. As figure ?? shows, we also investigate scenarios that replicate

\section{$39 ?$.}

${ }^{40}$ For a more detailed classificatory table see also Section A.2 of the On-line Appendix. 


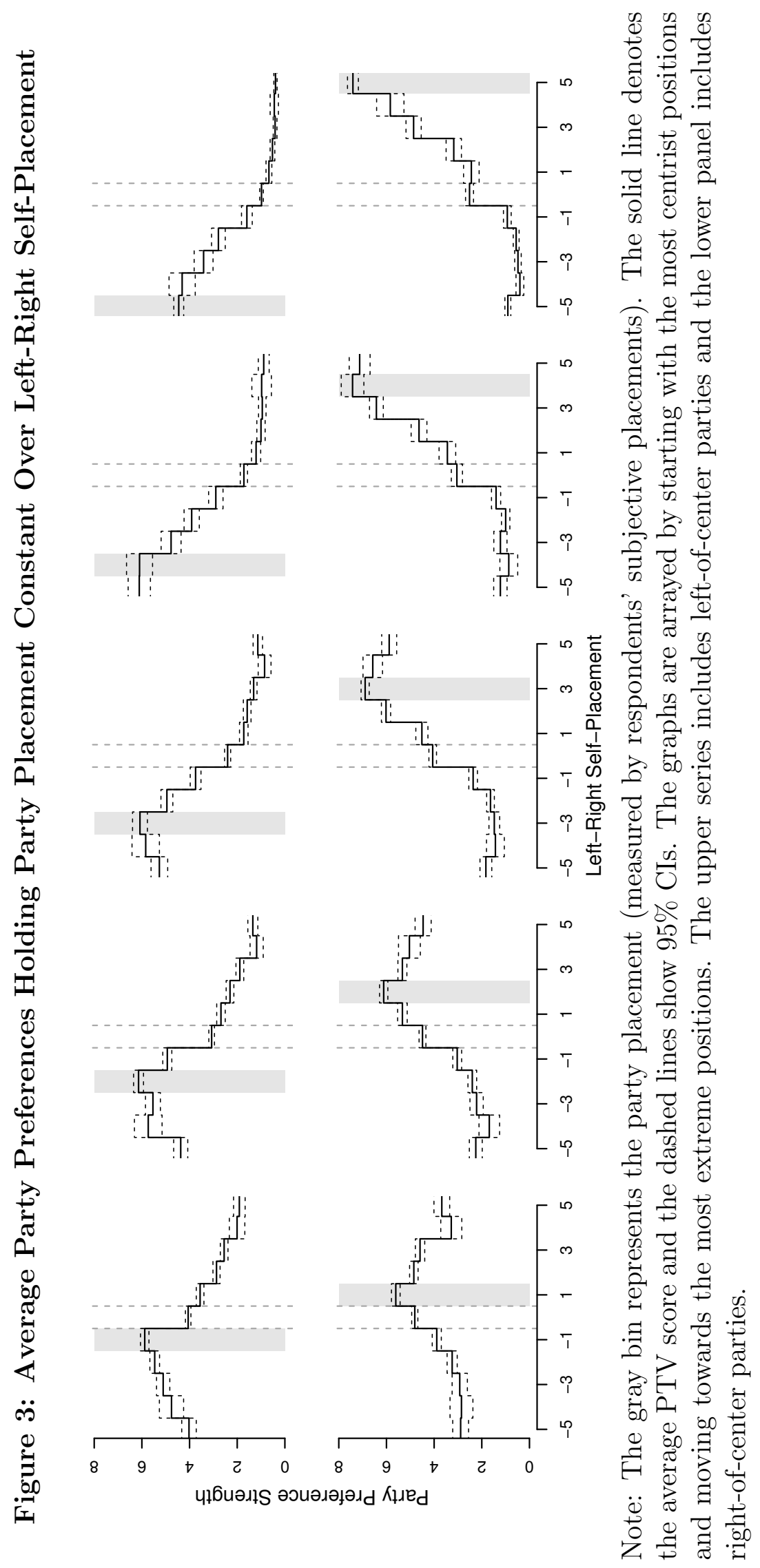




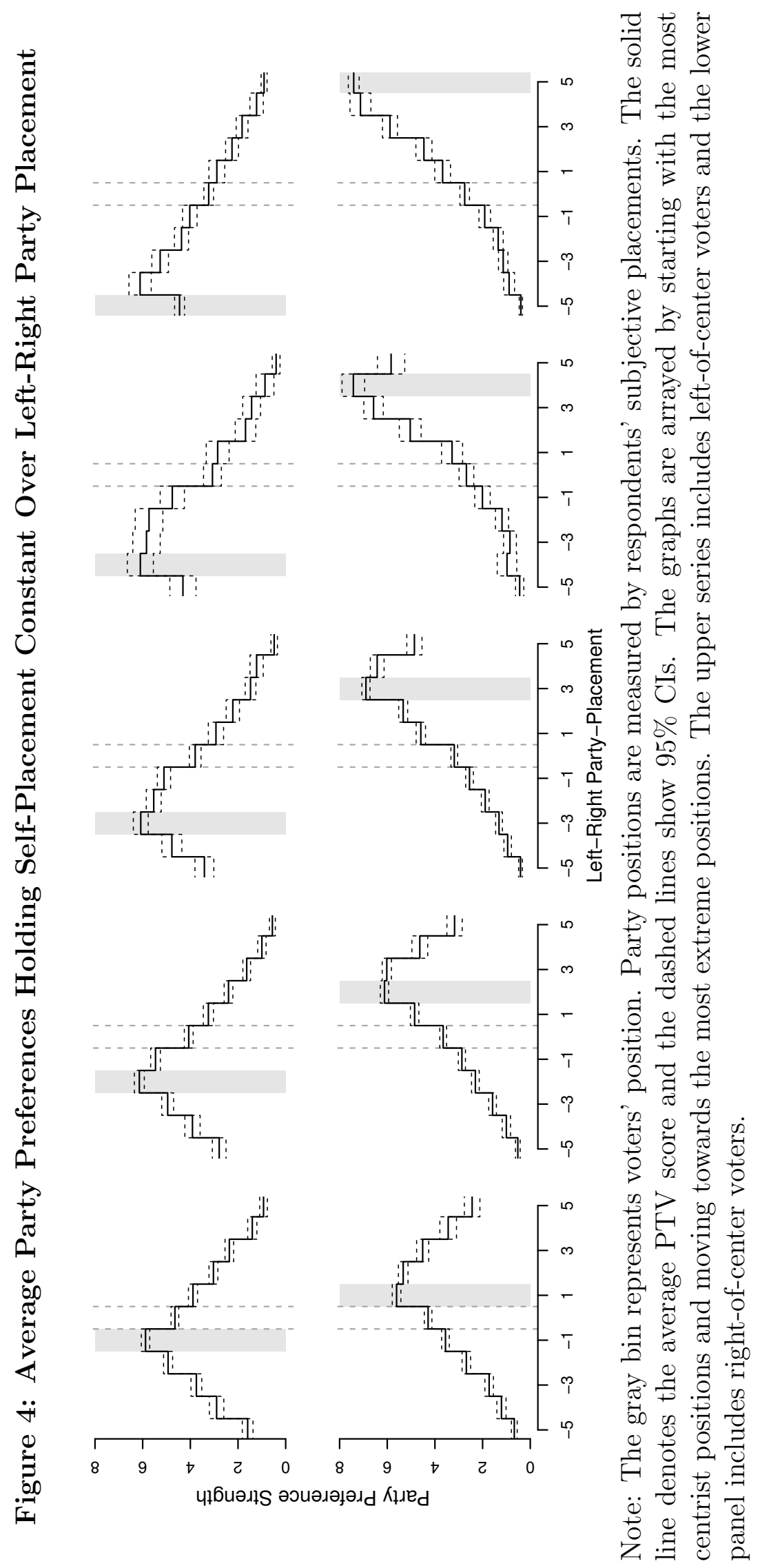




\section{Figure 5: Four Comparisons of Scenarios with and without Categorization}
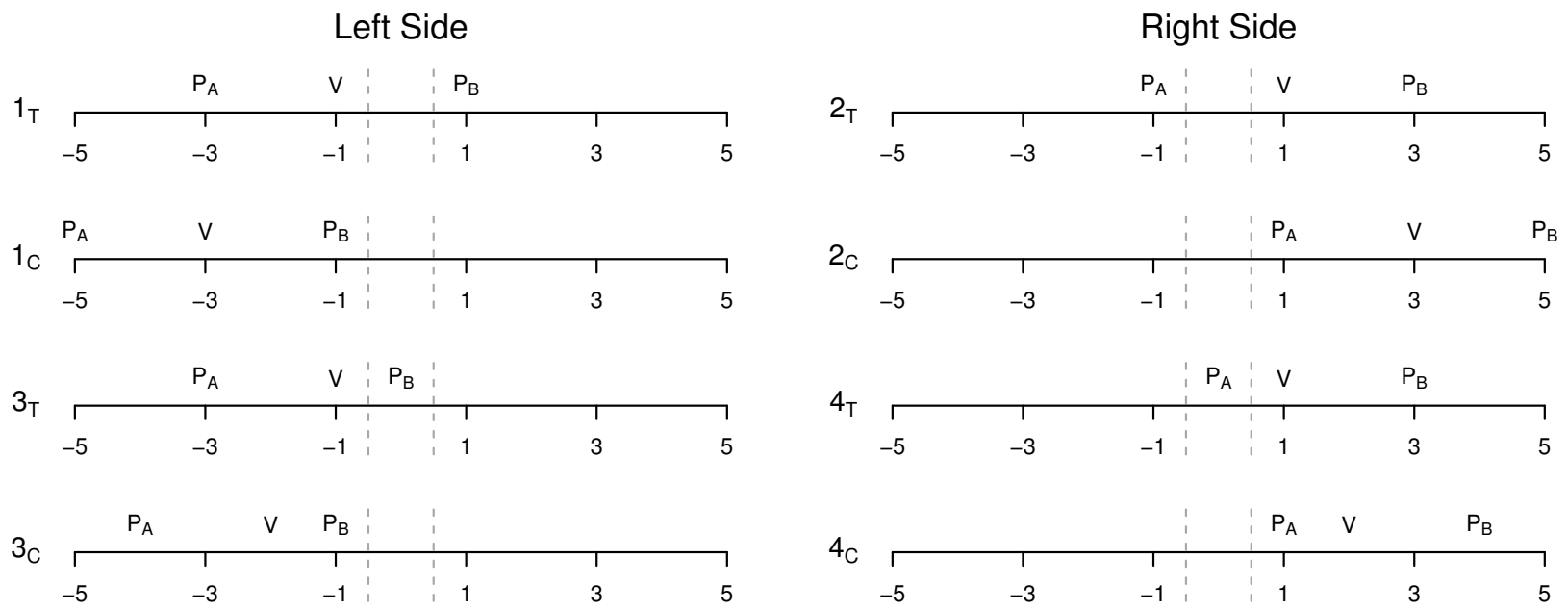

Note: The voter position is denoted $V$, while the positions of parties $A$ and $B$ are denoted $P_{A}$ and $P_{B}$. The comparisons are numbered 1 to 4 , and consist of a "Treatment" Scenario (where categorization implies a penalty for $P_{B}$ compared to $P_{A}$ ) and "Control" Scenario (where both parties are on the same side), subscripted $T$ and $C$, respectively.

the ones just presented, but on the right side of the scale (comparison 2). In comparisons 3 and 4 , we test the role of categorization, while letting the proximity of the two parties vary. In each of the scenarios involved, the proximity model predicts voter $V$ to prefer party $B$ over $A$, while directional theory predicts $A$ over $B$. However, the distances are kept constant from one scenario to the other, so proximity theory still predicts no difference between the two scenarios. The directional model yields the same prediction as before a negative difference-in-differences. According to the categorization model, there should be a positive difference-in-differences. However, these tests are more challenging for the categorization argument because the distinction is not between same versus opposite side but rather between same-side versus neutral status.

For each scenario, we select voters who place themselves at point $V$ and have located at least one party at $P_{A}$ and at least one party at $P_{B}$. We create a binary indicator identifying the alternative favored by the directional model (always party $A$ ). We then pool the observations for the two scenarios to be compared, create another binary indicator identifying the "treatment" scenarios, and interact this with the dummy identifying party $A$. The resulting 
coefficient denotes the difference in the average difference between the preference scores for party $A$ and party $B$, going from scenario $C$ to $T$. A negative coefficient would be consistent with directional theory, while a positive coefficient would be consistent with categorization theory. To account for the clustering of preferences within individuals, we include individual fixed-effects, and use robust standard errors, clustered at the individual level.

Figure ?? shows the results, plotting the coefficients of interest along with $95 \%$ confidence intervals. As can be seen, the point estimates are all positive; two are clearly significant, while one is barely significant, and one is barely insignificant. As explained above, these estimates are in line with the categorization model, while contradicting directional theory.

\section{Figure 6: Estimated Differences in Differences for Comparisons 1 to 4}

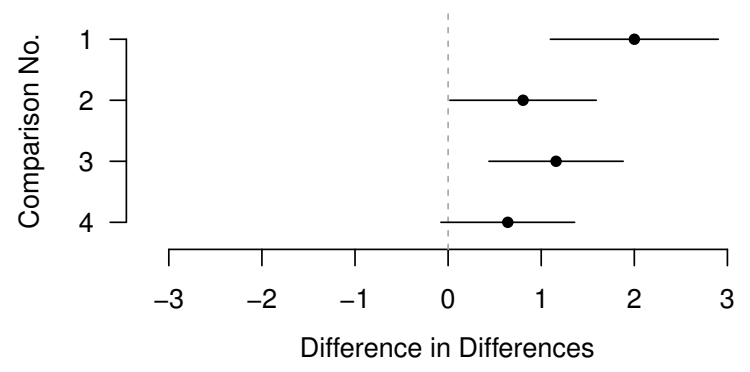

Note: The dots represent the difference in the average differences between the preference scores for party $A$ and party $B$, going from scenario $C$ to $T$. The error bars give $95 \%$ confidence intervals. The estimations include individual fixed effects and the standard errors are clustered at the individual level.

\section{Parametric Analysis}

To obtain more general estimates of the effects, we now turn to a more standard regression framework. Table ?? reports a set of regression models, implementing the categorization model along with other spatial functions. Model 1 includes the Proximity Term, the Directional Term, and indicators of Same Side and Opposite Side status. The estimated effect of Same Side is close to 1-point on the 11-point preference scale, while that of Opposite Side is about -.3 , and both estimates are highly significant. The effect of proximity is also highly significant and has the expected sign. The estimate for the Directional Term appears to 
be statistically significant, but it has the wrong sign, suggesting this model is misspecified. This conclusion is also corroborated by the plots shown above, which indicate no trace of a directional effect. The Directional Term is therefore dropped in Model 2, without a notable loss in explanatory power, or change in the other estimates. The categorization effects are still significant and having a considerable magnitude.

Table 1: Models of Spatial Voting with Categorization Effects

\begin{tabular}{lcccc}
\hline \hline & Model 1 & Model 2 & Model 3 & Model 4 \\
\hline Directional Term & $-0.027^{* * *}$ & & & \\
Proximity Term & $(0.003)$ & & & $-0.519^{* * *}$ \\
& $-0.572^{* * *}$ & $-0.500^{* * *}$ & & $(0.013)$ \\
Same Side & $(0.011)$ & $(0.009)$ & & $1.196^{* * *}$ \\
& $1.030^{* * *}$ & $0.933^{* * *}$ & $0.777^{* * *}$ & $(0.056)$ \\
Opposite Side & $(0.041)$ & $(0.040)$ & $(0.048)$ & $-0.885^{* * *}$ \\
& $-0.320^{* * *}$ & $-0.278^{* * *}$ & $-0.271^{* * *}$ & $(0.068)$ \\
Proximity Term & $(0.043)$ & $(0.042)$ & $(0.052)$ & $-0.210^{* * *}$ \\
$\quad$ & & & $(0.021)$ \\
Proximity Term & & & & $0.117^{* * *}$ \\
$\quad \times$ Opposite Side & & & & $(0.014)$ \\
Constant & $4.197^{* * *}$ & $3.910^{* * *}$ & $3.230^{* * *}$ & $4.017^{* * *}$ \\
& $(0.042)$ & $(0.039)$ & $(0.055)$ & $(0.045)$ \\
\hline Individual-Fixed Effects & Yes & Yes & Yes & Yes \\
Party-Fixed Effects & Yes & Yes & Yes & Yes \\
Proximity-Fixed Effects & No & No & Yes & No \\
\hline Adjusted $R^{2}$ & 0.398 & 0.396 & 0.286 & 0.399 \\
Observations & 94833 & 94833 & 52979 & 94833 \\
Individuals & 13278 & 13278 & 12302 & 13278 \\
\hline \hline
\end{tabular}

Note: Note: ${ }^{*} p<0.05,{ }^{* *} p<0.01,{ }^{* * *} p<0.001$. The dependent variable is the propensity to vote for a party (see the data section for details). Party positions are measured by respondents' subjective placements. The cell entries are OLS regression estimates, with robust standard errors, clustered at the individual-level, in parentheses.

There is one reason for concern with the reported results, however. Using the whole set of observations and a linear implementation of the Proximity Term, the analyses involve some extrapolation. All three side-statuses can only be observed for proximity values of 2,3 and 4. (It takes 2 steps to move from same to opposite side, while with a distance of 5 or larger, it is not possible to be on the same side. With a proximity value of 1 , it is only possible to 
compare either same or opposite side status to the neutral status.) In other words, there is only common support for the mentioned proximity values. Model 3 in Table ?? reports an analysis only including observations where the Proximity Term is $1,2,3$, or 4 . It further includes proximity-fixed effects, ensuring that proximity effects are fully controlled for. The resulting categorization estimates are only slightly weaker than before, with a Same Side estimate of about .8 and Opposite Side estimate of about -.3.

As discussed above, voters need more criteria than side categorizations to distinguish between several parties on a given side, and we expect these criteria to play a larger role for parties on the same side as voters, as the voters need to pick a winner of their vote. So far, our analyses show that when categorization in terms of sides does not suffice, voters apply a proximity logic to make finer distinctions. In Model 4, we therefore include an interaction between the Proximity Term and the categorization indicators. The results are in line with our expectations: The effect of the Proximity Term is almost twice as strong for parties on the same side as voters, compared to parties on the opposite side (-.73 versus -.40). Thus, categorization does not only create discontinuities in what proximity theory suggests is a continuous space, it also qualifies the impact of proximity considerations.

\section{Categorization Effects in Projection Bias}

We now turn to another implication of our theory. Rationalization or projection bias presents a considerable challenge for the proximity model, as it is built on the assumption that perceptions precede utilities. A key point for us is that if our theory is correct, projection should reflect the coarse categories examined above. A classic idealized model of projection considers the interaction between individuals' self-placements and their party evaluations. The more a voter likes a party, the more likely she is to perceive this party as being closer to her own position than it really is. As this process has been suggested as an alternative explanation of proximity patterns in voter preferences, it has invariably been assumed to operate in a linear fashion throughout the ideological scale. In contrast to this widespread 
assumption, the theory presented here implies categorization effects: The incentive to misplace a preferred party's true position is significantly strengthened when this means bringing it to one's own side. Thus, among two individuals with the same distance to their preferred party, projection should be more evident for the individual who would otherwise find the party at the opposite side of the center.

To test this hypothesis, we draw on, but also significantly modify, existing models of projection bias. ${ }^{41}$ We present two different models, both of which use parties' sample mean placements as a measure of their "true" positions. ${ }^{42}$ In our first model, the dependent variable, $W_{i j}$, is constructed as a binary indicator of "mis-categorization," i.e. party-individual combinations where the respondent placed the party at the opposite side of the sample average. Our measure of party utility, which is the same as before, is now employed as an independent variable, denoted $Y_{i j}$ (for individual $i$ and party $j$ ). In our simplest model, this variable is interacted with two binary indicators of same and opposite sides, $S_{i j}^{M}$ and $O_{i j}^{M}$, where mean party placements are used to locate the parties. The first indicator thus identifies observations where respondents are on the opposite side of the mean party placement whereas the second identifies those where respondents are on the same side as the mean party placement. The interaction between party evaluations and these two dummies reveals the impact of categorization-based projection: The more a voter likes a party on the opposite side, the more likely she is to misplace the party so as to find it on her own side. Our simplest model is thus as follows:

$$
W_{i j}=\beta_{0}+\beta_{1} O_{i j}^{M}+\beta_{2} S_{i j}^{M}+\beta_{3} Y_{i j}+\beta_{4} O_{i j}^{M} Y_{i j}+\beta_{5} S_{i j}^{M} Y_{i j}+\epsilon_{i j}
$$

${ }^{41}$ See ??.

${ }^{42}$ Sample mean placements have been shown to correlate very highly with other, more objective, measures of party positioning, such as those stemming from the coding of manifestos and expert surveys. See, for instance, ?. Nevertheless, as a robustness check, we have also implemented this analysis using party positions based on Chapel Hill expert surveys (?). The results are very similar with either approach. We present these results in the On-line Appendix (Section A.4). 
In this model, categorization effects in projection are mainly captured by $\beta_{4}$ and $\beta_{5}$, although these coefficients should be combined with the main effects of preference strength $\left(\beta_{5}\right)$ and side status (either $\beta_{1}$ or $\beta_{2}$ ) to obtain the complete marginal effects of preference strength, conditional on side status. We go one step further, however, and allow for nonlinearity by using penalized smoothing splines in a generalized additive model (GAM). We estimate three smooth terms, to capture the effect of preference strength, conditional on whether voters are on the same side as the mean party placement $\left(S_{i j}^{M}=1\right)$, opposite side $\left(O_{i j}^{M}=1\right)$, or neither $\left(S_{i j}^{M}=O_{i j}^{M}=0\right)$.

Our second model aims more directly to capture the underlying idea behind projection models, namely that voters might over- or under-estimate their ideological distance to parties according to their non-ideology-based preferences. Thus, the dependent variable is now a measure of divergence between the perceived and "objective" distances between parties and voters: $\left|P_{i j}^{P}-V_{i}\right|-\left|P_{j}^{M}-V_{i}\right|$, where $P_{j}^{M}$ denotes the mean left-right position of party $j$, $P_{i j}^{P}$ denotes the position of party $j$ as perceived by voter $i$, and $V_{i}$ denotes voter placements. We model this difference as a function of party utility $\left(Y_{i j}\right.$, proxied again with our PTV measure), the two indicators of same- and opposite-side ( $S_{i j}^{M}$ and $O_{i j}^{M}$, respectively), and the interaction between these and party utility. We expect that the need for rationalization will be stronger when preferred parties" "true" positions are on the opposite side of voters. ${ }^{43}$ Following the standard convention for first-differenced models, we also include the perceived ideological distance between parties and voters $\left(\left|P_{j}^{M}-V_{i}\right|\right)$ as a predictor in some of the models (reported in full in the On-line Appendix). The resulting model is thus:

$$
\left|P_{i j}^{P}-V_{i}\right|-\left|P_{j}^{M}-V_{i}\right|=\beta_{0}+\beta_{1}\left|P_{j}^{M}-V_{i}\right|+\beta_{2} O_{i j}^{M}+\beta_{2} S_{i j}^{M}+\beta_{3} Y_{i j}+\beta_{4} O_{i j}^{M} Y_{i j}+\beta_{5} S_{i j}^{M} Y_{i j}+\epsilon_{i j}
$$

We expect that the stronger the preference for the party, the greater the underestimation of the distance between party and voter. Such evidence for projection, however, should be

${ }^{43}$ Although we use mean party placement as an indicator of objective party positioning, in the On-line Appendix (section A.4) we also use the Chapel Hill placement with substantively identical results. 
mediated by whether the party is already on the same side as the voter. For a preferred party to be on the opposite side of the voter would make the need for projection stronger and thus increase the tendency for underestimation of ideological distance. Testing this expectation, we again use a generalized additive model to allow a more flexible estimation of the role of party utility for projection.

Figure ?? reports the results from both models. The first panel presents the key estimates for the first model and demonstrates considerable categorization effects in projection bias. The graph shows that when a voter strongly dislikes a party on her side $\left(Y_{i j}=0\right)$, the probability of miscategorizing the party (moving it to the opposite side) increases. This kind of misplacement becomes less likely as the evaluation of the party grows more favorable. Similarly, a voter strongly liking a party on the opposite side is much more likely to move this party to her own side. In contrast, if she dislikes a party on the opposite side, the probability of making such a miscategorization decreases considerably. ${ }^{44}$ The second panel shows that as party utility increases, the ideological distance to the party is increasingly underestimated. However, this effect is stronger for parties located at the opposite side than for parties on the same side as voters. The slope of $Y_{i j}$ is steeper for those parties that are on the opposite side. Taken together, these results strongly support the notion that voters categorize parties as well as themselves in terms of their side of the center, and that they find these categories significant. ${ }^{45}$

${ }^{44}$ In the On-line Appendix, we present all the estimation details together with various different model specifications. In particular, we also control for the absolute distance between voter and mean party placement as well as the interaction between this variable and party preference. We do not present these results in the main text because we fear the inclusion of mean perceived proximity might generate post-treatment bias, as it already incorporates part of the projection that stems from individuals' self-placements. We also replicate all modes using Chapel Hill party placements as a proxy for "true" party positions. The results are robust to all these qualifications.

${ }^{45}$ Details about the estimation are provided in the On-line Appendix (Section A.4), where we also report the results using the Chapel Hill data. 


\section{Figure 7: Categorization Effects in Projection Bias}
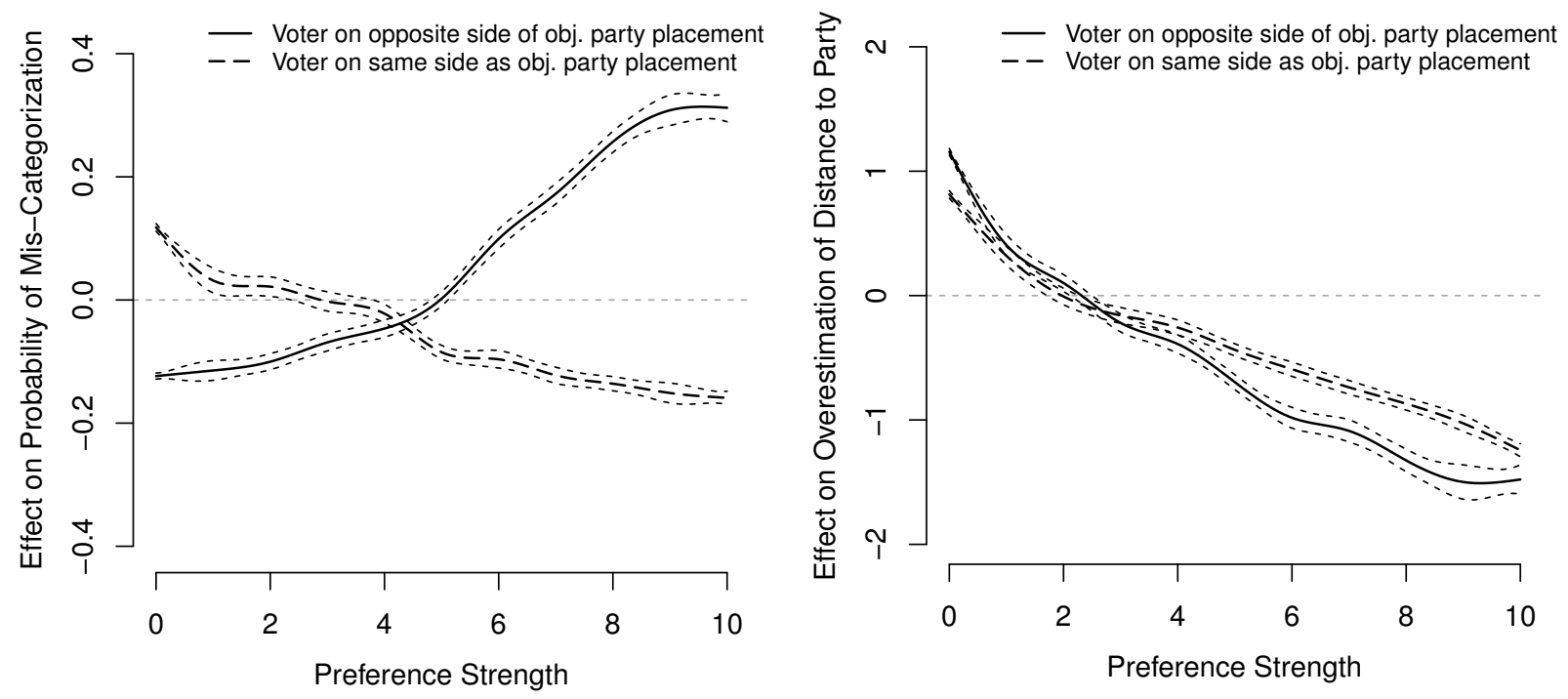

Note: The left panel shows the effects of party preference strength on the probability of placing a party on the "wrong side" compared to its sample mean placement, conditional on the mean party placement being on the same or opposite side of the voter. The right panel shows the effects of party preference strength the subjective overestimation of the distance to a party, conditional on the mean party placement being on the same or opposite side of the voter. Using Expert surveys to measure the party positions would give similar results. The plots are based on generalized additive models (GAM) with penalized smoothing splines. The dashed lines report $95 \%$ confidence intervals.

\section{Robustness}

Lastly, it worth assessing the robustness of our results. First, as many readers may be unfamiliar with our measure of party preferences, the "propensity to vote" (PTV), and some may consider actual vote choice a more important and relevant outcome, we also implement our model analyzing vote choice. In the appendix (Table A.6), we report a full replication of Table ?? with vote choice as the dependent variable, and the results are substantively identical to those we report above.

Another issue arises from the projection effects shown in the previous section: The question of whether the patterns we find are mainly driven by subjective perceptions (and misperceptions) of positions along the ideological scale, or whether they are still present when more objective measures are used. We address this using the Left-Right party positions pro- 
vided by the 2010 Chapel Hill expert survey. ${ }^{46}$ We implement our analyses for both PTVs and vote choice, and the results are reported in Table ??. ${ }^{47}$ The results are substantively very similar to those reported earlier, which implies that the categorization effects to a considerable degree are informed by objective party placements rather than being driven by individual misperceptions.

Table 2: Results with Party Positions Based on Chapel Hill Expert Surveys

\begin{tabular}{|c|c|c|c|c|}
\hline & Model 1 & Model 2 & Model 3 & Model 4 \\
\hline Dependent Variable & PTV & PTV & Vote & Vote \\
\hline Proximity Term & $\begin{array}{c}-0.465^{* * *} \\
(0.015)\end{array}$ & $\begin{array}{c}-0.451^{* * *} \\
(0.016)\end{array}$ & $\begin{array}{c}-0.022^{* * *} \\
(0.002)\end{array}$ & $\begin{array}{l}-0.015^{* * *} \\
(0.002)\end{array}$ \\
\hline Same Side & $\begin{array}{l}0.994^{* * *} \\
(0.054)\end{array}$ & $\begin{array}{l}1.107^{* * *} \\
(0.075)\end{array}$ & $\begin{array}{l}0.122^{* * *} \\
(0.006)\end{array}$ & $\begin{array}{l}0.180^{* * *} \\
(0.009)\end{array}$ \\
\hline $\begin{array}{l}\text { Proximity } \\
\quad \times \text { Same Side }\end{array}$ & & $\begin{array}{r}-0.045^{*} \\
(0.022)\end{array}$ & & $\begin{array}{c}-0.023^{* * *} \\
(0.003)\end{array}$ \\
\hline Constant & $\begin{array}{l}3.665^{* * *} \\
(0.064)\end{array}$ & $\begin{array}{l}3.583^{* * *} \\
(0.069)\end{array}$ & $\begin{array}{l}0.061^{* * *} \\
(0.008)\end{array}$ & $\begin{array}{l}0.041^{* * *} \\
(0.008)\end{array}$ \\
\hline Individual-Fixed Effects & Yes & Yes & Yes & Yes \\
\hline Party-Fixed Effects & Yes & Yes & Yes & Yes \\
\hline Adjusted $R^{2}$ & 0.295 & 0.295 & 0.213 & 0.214 \\
\hline Observations & 80707 & 80707 & 65137 & 65137 \\
\hline Individuals & 12403 & 12403 & 9754 & 9754 \\
\hline
\end{tabular}

Another potential concern is that our findings are confounded by partisan attachments. It is indeed likely that subjective perceptions of ideological proximity to some extent may be overestimated for parties with which voters strongly identify. However, the robustness checks above - using objective party placements - already guard against this possibly confounding effect, and show that our results hold in the absence of perception biases. Furthermore, it is also very likely that party identification is partly driven by ideological proximity, which is $46 ?$.

${ }^{47}$ In these analyses, we drop the category of opposite side, as no parties are positioned exactly in the center with these data, and one category thus suffices to distinguish the sides. Note also that the number of observations is somewhat reduced, because the expert survey data is missing for a few minor parties. 
why we generally do not include party identification in our models, as this would generate post-treatment bias. Nevertheless, as another robustness check, we replicate the analyses of Table ??, adding a measure of partisanship that captures both the direction and the intensity of partisan attachments. Full details are provided in the On-line Appendix (Table A.10), which also presents the results. All key findings are robust to the inclusion of party identification, suggesting that ideological categorizations constitute an independent and more general source of identification, generating effects beyond those that can be accounted for on partisan grounds.

A final question is whether categorization applies also to the American context. In this case, we would not focus on the left-right dimension, but on the conceptually similar liberal-conservative dimension. More importantly, the country's two party-system could render the ideological categorization of political actors superfluous, as party-based divisions could represent an equivalent and equally encompassing form of categorization. To address the question of whether ideological categorization is relevant in the US, adding predictive power beyond party identification, we conduct an analysis of vote choice using the American National Election Studies (ANES) and report the results in the Table A.11 in the On-line Appendix. ${ }^{48}$ We use all surveys since 1972, because since then respondents have been asked to place themselves and parties in a 1-7 liberal-conservative scale. We first replicate Table 1, showing that categorization effects are present also in the American context. We then add party identification as a control variable (despite the risk of post-treatment bias), and show that the categorization model has explanatory power beyond that of party identification. ${ }^{49}$

$48 ?$.

${ }^{49}$ That said, the conditioning role of sides appears less pronounced, especially when party identification is included: The effect of proximity on vote choice does not appear to increase significantly when parties and voters are on the same side. This finding is not particularly surprising because the two-party system generates less incentives for further differentiation among parties of the same side. 


\section{Conclusion}

Our results confirm that politics is understood in terms of sides, resulting in a discontinuous political space. Even when presented with seemingly continuous ideological scales, voters are conscious of their own side and evaluate parties accordingly, giving significantly higher ratings to the parties they consider to be on the same side as themselves. On average, we find party preferences to be about 1 point higher on an 11-point scale, when parties are on the same side as voters, and about .3 lower when they are on the opposite side. This finding has important implications that merit further elaboration.

In contrast to prior theoretical accounts that treated spatial voting as a building block of rational choice models of voting, ${ }^{50}$ categorization theory postulates that spatial voting involves a nontrivial identity-based element that makes voters perceive their political alternatives coarsely. As our results show, for a moderate left-wing voter, a strongly leftist party may be preferable to moderate right-wing party, even when distance alone would predict otherwise. This is not because the former advocates policy change in a leftist direction with higher intensity, as directional theory would suggest, but because one party fits the left as a reference category, while the other does not. The fact that projection bias is also characterized by side-specific discontinuities strengthens our identity-based interpretation of these effects. In this respect, our study joins the new social-identity based theories of party branding, which reconcile the Michigan conceptualization of party identification with attitudinal updating and partisan lability. ${ }^{51}$ By the same token, categorization theory points to the role of group-oriented thinking in spatial voting. This coarsened logic is only compatible with the principle of utility loss minimization - underpinning proximity theory - if utilities are themselves partly defined in more coarsened terms.

Our theory qualifies the proximity model in two ways: First, the coarse categories of

\footnotetext{
${ }^{50}$ E.g. ?

51 ?.
} 
sides lie beneath and cut across the political space, and second, the impact of the more finely grained distinctions afforded by proximity considerations is conditional on these basic categories. In other words, the categorization model does not refute the Downsian paradigm but shows that the smallest-distance criterion applies differently within the subspaces defined by the center of the ideological scale. The findings presented here thus give credence to new developments in decision making theories. A noteworthy model in this literature, which draws on the principles of bounded rationality, is characterized by a two-step "categorizethen-choose" logic. ${ }^{52}$ According to this scheme, agents first categorize and then choose alternatives within their preferred category, and we argue that vote choice is characterized by a similar two-stage process: At the first stage, voters identify parties satisfying the criterion of being on the same side, while at the second-stage, they use a more specific decision rule to choose among these options.

Apart from qualifying the theoretical underpinnings of proximity theory, categorization also informs the discussion about competing metrics of distance in proximity models. Two distance functions have been mainly used: Euclidean distances with quadratic utility penalizations $\left(\left[V_{i j}-P_{i j}\right]^{2}\right)$ and city-block distances, with linear utility penalizations $\left(\left|V_{i j}-P_{i j}\right|\right)$. Since they both provide the same preference ranking, the two models have been typically used inter-changeably. ${ }^{53}$ However, studies that have tried to compare the two metrics in terms of their explanatory power have provided overwhelming empirical evidence in favor of the city-block metric. ${ }^{54}$ In our own analyses, we also found that city-block proximity is a far better predictor of voter preferences than Euclidean metrics. Our model helps explain this finding: The Euclidean metric gives linear distance greater weight when it is least needed, i.e. when parties and voters are likely to be on opposite sides and linear distances tend to be large. Conversely, this metric gives linear distance the least weight when it is needed the ${ }^{52} ?$
${ }^{53} ?$
${ }_{54}$ 
most, i.e. when voters and parties are on the same side and linear distances tend to be small.

Research on directional theory is also affected by our argument. Our results suggest that when controlling for categorization, the intensity part of the directional model is no longer important in voter's party preferences. Accompanied by the more in-depth analyses presented in the On-line Appendix, our findings question the theoretical foundation of directional theory as a whole. We argue that voters apply categories defined by the center of the scale, rather than the policy status quo. This is important, as the directional model is commonly operationalized using the geometric center of survey scales as the neutral point, which increases the correlation between the directional measure and the categorization measures. While the directional model still fails to clearly capture the effects we find, prior estimates of directional effects may have benefited from the presence of categorization effects, in the absence of appropriate controls. In short, our results suggest that effects previously attributed to directional models are largely due to categorization effects.

Our results also have important practical implications. As a way to grasp the impact of categorization effects on parties' electoral fortunes, we simulate a scenario where they play no role. ${ }^{55}$ Among the voters placing themselves closest to the center $($ at $+/-1)$, we find that about $10-12 \%$ would vote differently in the absence of categorization. At the extremes of the scale, this share drops slightly below $5 \%$. On average, we find that between 5 and $6.3 \%$ of the voters would vote differently (an estimate that is pulled down by the voters in the center). While 5-6\% may seem modest, it may be a significant figure considering that election outcomes tend to be determined by the few who change their votes in systematic

${ }^{55}$ In particular, we subtract the predictions based on categorization effects from the observed party preferences. For each voter, we then reassess which party is the first preference (or which parties, if there are several). Next, we check whether the party last voted for in the national election is still among each voter's first preferences. We identify the voters whose counterfactual first preferences do not include the party last voted for, and calculate their share of the initial pool of voters. We further calculate percentile-based confidence intervals by repeating this procedure on 2000 bootstrap samples, clustered at the individual level, and stratified at the country level. 
ways. $^{56}$

Finally, the findings shed light on the dynamics of party competition. A common observation among students of party politics is the absence of leapfrogging: When parties change their position, they tend to do so within a limited range, maintaining the overall ranking along the policy dimension in question. Categorization theory adds a prediction to this pattern: That parties will stay on a given side of the center, as they need to convince their supporters that they remain on their side. This may account for the absence of leapfrogging, as it adds a boundary to party movement, and the parties around the center might otherwise have been the most likely to leapfrog each other. Even the most centrist parties tend to have a distribution of supporters that is skewed towards one side of the left-right continuum, and in light of the categorization model, this is likely to give these parties incentives to stay on the side of their traditional voters.

56 See ?, ch. 1 . 